\title{
Effect of Aerobic Exercise on Serum Adiponectin and Serum Malondialdehyde (MDA) In Type 1 Diabetic Patients
}

\author{
Mai Mohamed Elbalshy, Mariam Elsayed Mohamed, Awny Fouad Rahma \\ Department of Physical Therapy for Internal Medicine, Faculty of Physical Therapy, Cairo University, Cairo, Egypt \\ Email address: \\ dr_hasaballah@yahoo.com (M. M. Elbalshy) \\ To cite this article: \\ Mai Mohamed Elbalshy, Mariam Elsayed Mohamed, Awny Fouad Rahma. Effect of Aerobic Exercise on Serum Adiponectin and Serum \\ Malondialdehyde (MDA) In Type 1 Diabetic Patients. European Journal of Preventive Medicine. Vol. 5, No. 5, 2017, pp. 71-76. \\ doi: 10.11648/j.ejpm.20170505.14
}

Received: August 4, 2017; Accepted: August 21, 2017; Published: September 18, 2017

\begin{abstract}
Type 1 diabetes is fatal unless treated with insulin. Exercise interventions have been shown to be as efficient as the pharmaceutical agents to improve glycaemic control. The aim of the current study was to determine the effect of aerobic exercise on possible mechanisms controlling insulin resistance. Forty diabetic type 1 patients of both sexes (20 females and 20 males) were participated in the study. They were randomly divided into two equal groups. Study group comprised of 20 patients participated in a supervised moderate-intensity aerobic exercise program performed on bicycle ergometer for 50 minutes, 3 times per week, for 8 weeks, in addition to their medical treatment while the control group received only their medical treatment. The result showed that a significant decrease in insulin resistance in the Study group as compared to the control group. The mean values of adiponectin were significantly increased from $5.85 \pm 0.65$ to $6.6 \pm 0.56$ in the study group. The mean values of Malondialdehyde (MDA) and fasting glucose were significantly decreased from 7.2 $\pm 0.98,146.88 \pm 11.42$ to $6.45 \pm 0.95,133.87 \pm 11.99$ respectively in the study group. It can be concluded that aerobic exercise has a positive effect on decreasing insulin resistance and hence decrease the incidence of diabetic complications.
\end{abstract}

Keywords: Type 1 Diabetes' Insulin, Aerobic Exercise, Insulin Resistance

\section{Introduction}

Diabetes mellitus, often simply referred to a group of metabolic diseases in which a person has high blood sugar, either because body does not produce enough insulin, or because cells do not respond to the insulin that is produced [1]. It is a disorder of glucose metabolism and is classified into multiple types based on underlying pathophysiology. The two most common types of diabetes are type 1 and type 2. Type 1 diabetes mellitus (T1DM) is characterized by an absolute deficiency of insulin, and is seen most often in children and adolescents. The classical symptoms are polyuria (frequent urination), polydipsia (increased thirst), polyphagia (increased hunger), and weight loss [2].

Successful model of exercise can control type 1 diabetic patients. It reduces hospital admissions, improves diabetic control, and improves quality of life scores. Patients may be unwilling or unable to be accountable for health care coordination. The development of exercise policies and the adjustment in financial rewards to health care organizations will have a major impact in implementing these programs over the next 10 years. [3].

Adiponectin known as a gelatin binding protein-28 (GBP$28)$ is an adipose tissue-specific collagen-like molecule described in 1996 and expressed exclusively in adipocytes. It is present in human circulation in high levels $(5-30 \mathrm{mcg} / \mathrm{ml})$ accounting for $0.01 \%$ of plasma proteins, with slightly higher levels in women than men. It has been shown to have antidiabetic, anti-atherosclerotic and anti-inflammatory functions. Studies provide promising results for diagnostic and therapeutic role of adiponectin in obesity, insulin resistance and diabetes [4].

Oxidative stress products do not originate exclusively in the lungs, as they are formed in situ from the phospholipids of the cellular membranes and lipoproteins. After they are released to extracellular space, they circulate in the plasma and may be detected in urine. Oxidative stress is a phenomenon associated with pathogenetic mechanisms of several diseases, including atherosclerosis, cancer, diabetes mellitus and inflammatory diseases, as well as with aging 
processes. The levels of oxidative stress markers in plasma and urine, especially of 8-isoprostane and MDA reflect systemic production in diseases such as diabetes, hypertension and renal diseases [5].

Malondialdehyde (MDA) is a major player in low density lipoprotein (LDL) modification and is a product of the peroxidation of arachidonic, eicosapentaenoic and docosahexaenoic acids. The pathologic effects of LDL include the induction of atherosclerosis (by stimulating monocyte infiltration and smooth muscle cell migration and proliferation), atherothrombosis (by inducing endothelial cell apoptosis), and plaque erosion (by impairing the endothelial anticoagulant balance). Although microvascular and macrovascular complications of DM are known to increase with DM duration [6, 7].

Few longitudinal studies showed the preventive effect of fitness on metabolic syndrome [8]. Exercise interventions have been shown to be as efficient as the pharmaceutical agents to improve glycaemic control. Aerobic exercise has positive benefits for those who have type 1 diabetes. It can lower blood sugar levels, improve insulin sensitivity, strengthen the heart, lowers blood pressure, improves cholesterol levels, and decreases body fat $[9,10]$.

Exercise is an effective non-pharmacological treatment to increase adiponectin level and decrease risks of ischemic heart disease. Although the basic mechanisms involved in changes in adiponectin levels through exercise have not yet been fully understood, negative energy balance appears to be more effective in adiponectin increase [11].

Acutely, in response to a single bout of exercise, a proinflammatory response is generated (increase in circulating leukocytes and in systemic concentrations of pro-inflammatory cytokines and chemokines), whose damaging potential is limited by simultaneous activation of anti-inflammatory mechanisms. Conversely, repeated exercise training results in significant reduction of the systemic inflammatory state [12]. It has an important role on the decrease of oxidative stress parameter and thus it may has a possible protective effect against oxidative stress in diabetics [10]

Thus, the results of studies on the effects of aerobic training on adiponectin and Malondialdehyde (MDA) still controversy. Therefore the purpose of this study was to investigate whether 8 weeks of aerobic training would affect serum adiponectin and MDA in patients with type 1 diabetes mellitus which can, not only improve glycemic control, but also substantially improve capacity for independent living and enhanced quality of life.

\section{Materials and Methods}

\subsection{Subjects Characteristics and General Experimental Design}

Study Subjects: forty patients with type 1 diabetes were recruited from the outpatient-clinic of internal medicine of Brket El-Sabh General Hospital with age ranged from 25 to 35 years old and body mass index (BMI) ranged from 30 to
$34.9 \mathrm{~kg} / \mathrm{m}^{2}$ (class I obesity). They diagnosed as diabetic patients before the age of 30 with fasting glucose level ranged from $6.2-9.5 \mathrm{mmol} / \mathrm{L}$ and only on insulin medications use being required within the first year of diagnosis and/or diabetic ketoacidosis presence or a trend ketosis, they were free from cardiovascular and respiratory problems such as unstable angina, myocardial infarction and chronic chest diseases as well as free from autonomic neuropathy and orthopedic problems. They were not participated in any form of regular physical activity and were instructed not to make any diet throughout the study.

\subsection{Evaluated Parameters}

\subsubsection{Anthropometric Measurements}

Weight and height were measured by Standard height and weight scale (floor type model; ZT-120, made China) to measure BMI. In indoor clothing without shoes, and BMI was calculated as weight $(\mathrm{kg}) /$ height $\left(\mathrm{m}^{2}\right)$ [13].

\subsubsection{Laboratory}

Disposable plastic syringes were used to draw $3 \mathrm{ml}$ of venous blood samples and polypropylene tubes with EDTA to keep blood samples. Serum Adiponectin level and serum malondialdehyde (MDA) were measured by ELISA method also fasting serum glucose was measured.

Subjects were assigned randomly into two groups equal in number: Study group: fifteen matching patients were participated in a supervised moderate-intensity aerobic exercise program performed on bicycle ergometer for 50 minutes, 3 times per week, for 8 weeks, in addition to their medical therapy, Control group: fifteen matching patients were received their medications only. All sessions were supervised and participation assessed. All patients were free to withdraw from the study at any time. All participants provided their informed consent after receiving a detailed explanation of the study. The data of all the patients were available.

\subsection{Treatment Procedures Moderate-Intensity Aerobic Exercise Program}

\subsubsection{Mode of Training}

Cycle ergometer was used in the aerobic training of the patients in the study group.

\subsubsection{Intensity and Duration of Training}

The study group was undergone 50 minutes of aerobic training with a target heart rate of $65 \%$ of their age-predicted maximum heart rate [14].

Frequency of training:

Three times a week for eight weeks with a total of 24 sessions.

\subsubsection{Training Session Will Consist of}

A) The warming up: consists of 5-10 minutes pedaling on the cycle ergometer at low speed.

B) The active training phase: 30 minutes with a target heart rate of $65 \%$ of their age-predicted maximum heart rate. 
C) The cool down: consists of 5-10 minutes pedaling at low speed.

Table 1. Descriptive statistics for comparing the mean age, weight and height of study and control groups.

\begin{tabular}{lllll}
\hline Groups & Study group & Control group & P-Value & Sig \\
\hline Age (years) & $29.2 \pm 5.02$ & $29.2 \pm 5.47$ & 1.00 & NS \\
Weight (kg) & $87.35 \pm 7.88$ & $91.65 \pm 9.83$ & 0.135 & NS \\
Height $(\mathrm{cm})$ & $164.25 \pm 7.36$ & $164.35 \pm 8.59$ & 0.969 & NS \\
\hline
\end{tabular}

Age-predicted maximum heart rate $=220$-age [15]

\subsubsection{The Cool Down}

Consists of 5-10 minutes pedaling at low speed.

\subsection{Statistical Analysis}

The mean values of serum adiponectin, serum malondialdehyde (MDA) and fasting serum glucose obtained before and after 8 weeks of training program. All these findings allowed the researchers to conduct parametric analysis. Accordingly, $2 \times 2$ mixed design MANOVA was used to compare the tested variables of interest at different tested groups and measuring periods $(\mathrm{P}<0.05)$.

\section{Results}

The study involved forty patients of both sexes with type 1 diabetes. Their age ranged from 25 to 35 years. The patients were assigned into two groups equal in number: the first group (study group) received a supervised moderate-intensity aerobic exercise program performed on bicycle ergometer for 50 minutes, 3 times per week, for 8 weeks, in addition to their medical therapy. The second group (control group) received their medications only in order to investigate the effect of 8 weeks of aerobic training on serum adiponectin, MDA and fasting glucose in patients with type 1 diabetes mellitus. (Table 1) represented non significance difference between both groups in age, weight and height. The mean values of adiponectin were significantly increased from $5.85 \pm 0.65$ to $6.6 \pm 0.56$ in the study group and from $6.49 \pm 0.71$ to $6.44 \pm 0.73$ in control group (Table 2). The mean values of Malondialdehyde (MDA) and fasting glucose were significantly decreased from $7.2 \pm 0.98,146.88 \pm 11.42$ to $6.45 \pm 0.95,133.87 \pm 11.99$ respectively in the study group and from $6.65 \pm 0.81,139.7 \pm 13.53$ to $6.62 \pm 0.85,138.05 \pm 14.56$ in control group (Table 3 and table 4). So, it can be concluded that eight weeks of aerobic exercises have a positive effect on serum adiponectin, MDA and fasting glucose in patients with type 1 diabetes mellitus which reflect a positive effect on decreasing insulin resistance and hence decrease the incidence of diabetic complications.

\section{Discussion}

The current study was conducted to determine the effect of aerobic exercise on possible mechanisms controlling insulin resistance. The mean values of adiponectin were significantly increased in the study group and also mean value of
Malondialdehyde (MDA) and fasting glucose were significantly decreased in the study group. This means that in

Table 2. Mean value and significance of adiponectin pre and post study at both groups.

\begin{tabular}{lllll}
\hline Adiponectin mg/dl & Pre- study & Post- study & MD & P-value \\
\hline Study group & $5.85 \pm 0.65$ & $6.6 \pm 0.56$ & 0.75 & 00.0001 \\
Control group & $6.49 \pm 0.71$ & $6.44 \pm 0.73$ & 0.05 & 0.205 \\
\hline
\end{tabular}

Table 3. Mean value and significance of malondialdehyde (MDA) pre and post study at both groups.

\begin{tabular}{lllll}
\hline $\begin{array}{l}\text { Malondialdehyde } \\
\text { Jnj, (MDA) }\end{array}$ & Pre- study & Post- study & MD & P-value \\
\hline Study group & $7.2 \pm 0.98$ & $6.45 \pm 0.95$ & 0.755 & 00.0001 \\
Control group & $6.65 \pm 0.81$ & $6.62 \pm 0.85$ & 0.03 & 0.425 \\
\hline
\end{tabular}

Table 4. Mean value and significance of fasting glucose pre and post study at both groups.

\begin{tabular}{lllll}
\hline fasting glucose & Pre- study & Post- study & MD & P-value \\
\hline Study group & $146.88 \pm 11.42$ & $133.87 \pm 11.99$ & 13.005 & 00.0001 \\
Control group & $139.7 \pm 13.53$ & $138.05 \pm 14.56$ & 1.655 & 0.023 \\
\hline
\end{tabular}

patients with type 1 diabetes mellitus, aerobic exercise have a positive effect on improving adiponectin, MDA and fasting glucose which decrease insulin resistance and decrease tendency of diabetic complications.

Malondialdehyde (MDA) is an important marker which may reflect low-grade systemic inflammation. MDA is produced in various tissues under diabetic conditions and has often been implicated in the pathogenesis of micro and macro vascular diseases observed in diabetic individuals. An association between physical inactivity and low grade systemic inflammation has been demonstrated in cross sectional studies [16].

Plasma levels of adiponectin inversely correlates with body mass index (BMI), intra-abdominal fat, and indices of insulin resistance. Many studies suggested that adiponectin is an important regulator of insulin sensitivity and glucose homeostasis and several studies have established an inverse relationship between the amount of physical activity and proinflammatory cytokines in obesity, diabetes, and the metabolic syndrome. Many believe that the beneficial effect of exercise is partly mediated through changes in the adipokines profile by increasing anti-inflammatory cytokines and decreasing proinflammatory ones. Exercise increases insulin sensitivity through reduction of resting levels of TNF$\alpha$ and CRP and augmentation of adiponectin levels [17].

Exercise has been accepted and generally recommended for the management of type 1 diabetes mellitus (T1D) and for improving the overall quality of life in affected individuals. In addition to increasing aerobic fitness, reducing cardiovascular risk factors, and reducing bodyweight and body fat, physical activity develops and maintains chronic glycemic control by enhancing insulin sensitivity and stimulating muscle glucose uptake. The American College of Sports Medicine (ACSM) has published a guideline for exercise testing and prescription in T1D which recommends that individuals with T1D need to work out for 20-45 
minutes at an intensity of $40-60 \%$ of their maximal oxygen consumption (VO2max) for 5-7 days/week, or daily at low to moderate intensity [18].

In current study two patients developed acute mild hypoglycemic attacks during the exercise treated with nutritional support. Their glucose level reached 4.7 and 4.4 $\mathrm{mmol} / \mathrm{l}$ respectively. Both patients were evaluated at the time of the glycemic attack with the physician on call. Their current session was cancelled and they continued their program with more close observation.

The current study was early supported by Svensson and Eriksson [19] which indicates that the change in the amount of body fat contributes to the change in insulin resistance over time in T1D patients and on theother hand, in another study, strength training has enhanced insulin sensitivity and improved glucose tolerance.

Sundell [20], Colberg et al [21] and Shakil-Ur-Rehman [22] agree with current results (a combination of strength and aerobic training) showed that there was a significant improvement in chronic glycemic control compared with a no exercise group. A possible explanation for this is the combined effect of a greater use of glucose, caused by an increased muscle mass and the decreased insulin resistance.

In agreement with our study, ARSLAN et al [15] who concluded that 12-week, aerobic-training program, without a concomitant weight loss diet, was associated with significant decrease in MDA levels in diabetic individuals and Michael et al [21] reported that exercise improves insulin resistance and glucose tolerance, and these adaptations may, in part, be related to reductions in inflammation and oxidative stress.

Gordon et al [10] agree with our study and suggested that Hatha yoga exercise and conventional physical therapy exercise may have therapeutic preventative and protective effects on diabetes mellitus by decreasing oxidative stress and improving antioxidant status. The control of glycaemia and the decreased lipid profile parameters using aerobic exercise are important influences on the decrease of this oxidative stress parameter and provide more support for the rational of a possible protective effect of aerobic exercise against oxidative stress in diabetics.

Rector et al [22] found that diet and exercise induced weight loss $(10 \%)$ improved measures of insulin sensitivity and beneficially altered biomarkers of oxidative status. In another agreement with our study, Ibanez et al [23] concluded that regular exercise can strengthen antioxidant defenses and may reduce oxidative stress.

In agreement with our results supervised exercise program for 10 weeks significantly improved insulin sensitivity and increased adiponectin levels in healthy subjects Lim et al [24]. Furthermore, 2 weeks of long skiing exercise resulted in increased adiponectin levels in healthy men Eriksson et al [25].

Study of plasma adiponectin levels in inactive, abdominally obese men showed that both acute and short term (one week) aerobic exercise training significantly increased plasma values Saunders et al [26] and another study who concluded that aerobic training program for 3 months leads to increased plasma adiponectin levels with a significant reduction in body fat mass without changes in body weight.

The results of the current study come in agreement with Oberbach et al [27] who conducted a 4-week program including swimming exercise alternating with running or biking, resulted in improvement in insulin sensitivity and plasma adiponectin concentrations in patients with IGT and T2DM, but not in the normal subjects and another human study revealed that 4 weeks of aerobic exercise training increased adiponectin concentration and AdipoR1 expression in the skeletal muscles of the subjects with IGT and T2DM as well as the normal subjects Bluher et al [28].

The duration of the training period is an important influencing factor for decreasing blood glucose levels. Blood glucose levels decreased significantly only in training studies that lasted for more than 3 months. While blood glucose levels are inversely correlated with the duration (minutes) of the exercise training, the amount (times/week) of training per week can also influence the blood glucose levels. Besides this, baseline glycemic control is also an important predictor of blood glucose improvement due to training. Blood glucose decreases significantly more in T1D individuals with poor glycemic control compared with individuals with good glycemic control Tonoli et al [18]

Lehman et al [29] demonstrated only a slight decrease in blood glucose in well controlled subjects who performed exercise training. This might suggest that exercise can be beneficial in order to maintain a good glycemic control in T1D subjects. Cardiopulmonary training adaptations are greater in patients with T1D who maintain good glycemic control Baldi et al [30]

Aerobic and strength training have different actions in the body and can therefore influence glycemic control through different pathways, for example, fat mass decrease after a period of aerobic training Ismail et al [31].

In disagreement with our results, acute episodes of mild or moderate exercise in healthy, lean subjects do not affect adiponectin levels Bobbert et al [32].

The results of current study revealed a significant reduction in insulin resistance and serum MDA, with a significant increase in serum adiponectin in the study group compared with the control group. There was a non-significant change in BMI in both groups at the end of the study.

These results demonstrated that aerobic exercise may improve insulin resistance and this improvement may be mediated, at least in part, by the increase in serum adiponectin and the decrease in serum MDA, without a significant change in BMI. So, aerobic exercise is recommended as an effective therapeutic tool which helps in controlling plasma glucose level and preventing complications in type 1 diabetic patient.

\section{Conclusion}

The results of this study support the importance of exercise training program on type 1 diabetic patients and showed that 
aerobic exercise has a positive effect on decreasing insulin resistance and hence decrease the incidence of diabetic complications. Also, the results of this study demonstrated that the improvement in insulin resistance may be mediated by the increase in adiponectin level and the reduction in MDA, independent of change in BMI, after aerobic exercise program.

In summary, it can be concluded that individuals with type 1 diabetes mellitus who follow this exercise program may benefit regarding improved insulin resistance, in addition to the beneficial effects of increased adiponectin (insulin sensitizer, anti-inflammatory, and antiatherogenic) and reduced MDA (oxidative stress marker) which can, not only improve glycemic control, but also substantially improve capacity for independent living and enhanced quality of life.

\section{References}

[1] Shoback, 2011. Greenspan's basic \& clinical endocrinology (9th ed.). New York: McGraw-Hill Medical. pp. Chapter 17. ISBN 0-07-162243-8.

[2] Silpa Nadella, Justin A. Indyk, and Manmohan K. Kamboj. Management of diabetes mellitus in children and adolescents: engaging in physical activityTransl Pediatr. 2017 Jul; 6(3): 215-224. doi: $10.21037 / \mathrm{tp} .2017 .05 .01$.

[3] Allison RL. 2017. Back to Basics: The Effect of Healthy Diet and Exercise on Chronic Disease Management. S D Med. 2017 Spec; No: 10-18

[4] Ziemke F and Mantzoros C. S., 2010. Adiponectin in insulin resistance: lessons from translational research. Am J Clin Nutr., 91(1): 258S-261S.

[5] Daniela P., Zdenka F., Kamila S., Stěpánka V., Jindřiška L., Ondřej P., Jaromír B., Tomáš N., Marek K. and Petr K., 2011. Oxidative Stress Markers in Exhaled Breath Condensate in Lung Fibroses Are NotSignificantly Affected by Systemic Diseases Industrial Health., 49, 746-754.

[6] Deshpande A. D., Harris-Hayes M. and Schootman M., 2008. Epidemiology of diabetes and diabetes-related complications. Phys Ther., 88: 1254-64.

[7] Nakhjavani M., Esteghamati A., Nowroozi S., Asgarani F., Rashidi A. and Khalilzadeh O., 2010. Type 2 diabetes mellitus duration: an independent predictor of serum malondialdehyde levels. Singapore Med J., 51(7): 582-5.

[8] Laura A. Crist, Catherine M. Champagne, Leonor Corsino, Lillian F. Lien, Guangyu Zhang and Deborah Rohm Young., 2012. Influence of Change in Aerobic Fitness and Weight on Prevalence of Metabolic Syndrome. Prev Chronic Dis., 9: E68.

[9] Sigal R. J., Kenny G. P., Boule N. G., Wells G. A., Prud'homme D. and Fortier M., 2007. Effects of aerobic training, resistance training, or both on glycemic control in type 2 diabetes. Annals of Internal Medicine., 147: 357-369.

[10] Gordon L. A., Morrison E. Y., McGrowder D. A., Young R., Fraser Y. T. P., Zamora E. M., Alexander-Lindo R. L. and Irving R. R., 2008. Effect of exercise therapy on lipid profile and oxidative stress indicators in patients with type 2 diabetes. BMC Complementary and Alternative Medicine., 8: 21.
[11] Parsian H., Eizadi M., Khorshidi D. and Khanali F., 2013. The effect of long-term aerobic exercise on serum adiponectin and insulin sensitivity in type 2 diabetic patients. Journal of Jahrom University of Medical Sciences., 11 (1): 36-43.

[12] Ounis O. B., Elloumi M., Zouhal H., Makni E., Denguezli M., Amri M., Lac G. and Tabka Z., 2010. Effect of Individualized Exercise Training Combined with Diet Restriction on Inflammatory Markers and IGF-1/IGFBP-3 in Obese Children. Ann Nutr Metab., 56: 260-266.

[13] Ferreira P. A., et al., Safety of the Six-Minute Walk Test in Hospitalized Cardiac Patients. International Journal of Cardiovascular Sciences., 2011, 28(1): 70-77.

[14] Ng C. L. W., Goh S. Y., Malhotra R., Ostbye T. And Tai E. S., 2010. Minimal difference between aerobic and progressive resistance exercise on metabolic profile and fitness in older adults with diabetes mellitus: a randomized trial Journal of Physiotherapy., 56: 163-70.

[15] Richer MJ, Miiger k, Tello k, Stlle P Seeger W, Mayer E, Ghofran AH., 2016. Heart rate response during 6-minute walking testing predicts outcome in operable chronic thromboembolic pulmonary hypertension., BMC Pulm Med $2016 \mathrm{Jul}$ 4: 16(1): 96.

[16] Mihriban A., HilmiIpekci s., Kebapcilar L., Dogan Dede N., Kurban s., Erbay E. and Sait Gonen M., 2014. Effect of Aerobic Exercise Training on MDA and TNF- $\alpha$ Levels in Patients with Type 1 Diabetes Mellitus. International Scholarly Research Notices., 2014: 1-5.

[17] Golbidi S. and Laher I., 2014. Exercise Induced Adipokine Changes and the Metabolic Syndrome Journal of Diabetes Research., 2014, 1-16.

[18] Tonoli Cajsa, Elsa Heyman, Bart Roelands, Luk Buyse, Stephen S. Cheung, Serge Berthoin and Romain Meeusen., 2012. Effects of Different Types of Acute and Chronic (Training) Exercise on Glycaemic Control in Type 1 Diabetes Mellitus A Meta-Analysis. Sports Med., 2012: 42 (12): 10591080 .

[19] Svensson M. K., Eriksson J. W., 2011. Change in the amount of body fat and IL-6 levels is related to altered insulin sensitivity in type 1 diabetes patients with or without diabetic nephro- pathy. Horm Metab Res., 43 (3): 209-15 87.

[20] Sundell J., 2011. Resistance training is an effective tool against metabolic and frailty syndromes. Adv Prev Med., 2011, $1-7$.

[21] Colberg SR, Sigar RJ, Yardley JE, et al. Physicalactivity/Exercise and Diabetes: A Position Statement of the American Diabetes Association. Diabetes Care 2016; 39: 2065-79. 10.2337/dc16-1728.

[22] Shakil-Ur-Rehman S, Karimi H, Gillani SA. Effects of supervised structured aerobic exercise training program on fasting blood glucose level, plasma insulin level, glycemic control, and insulin resistance in type 2 diabetes mellitus. Pak J Med Sci. 2017 May-Jun; 33(3): 576-580. doi: 10.12669/pjms.333.12023

[23] Michael A., Jon C., Edward P., Holly E., Jessica T., Venket R., Stephen G., George B., Maria R., Adria G., Mladen V. and Michael C., 2010. Exercise maintains euglycemia in association with decreased activation of c-Jun NH2-terminal kinase and serine phosphorylation of IRS-1 in the liver of ZDF rats. Am J Physiol Endocrinol Metab., 298: 671-682. 
[24] Rector S., Shana O., Ying L., Pamela S., Grace Y., Richard H., Craig S., Harold L., Kevin C. and Tom R., 2007. Exercise and diet induced weight loss improves measures of oxidative stress and insulin sensitivity in adults with characteristics of the metabolic syndrome. Am J Physiol Endocrinol Metab., 293: 500-506.

[25] Ibanez J., Izquierdo M., Arguelles I., Forga L., Larrion J. L., Garcia-Unciti M., Idoate F. and Gorostiaga E. M., 2005. Twice weekly progressive resistance training decreases abdominal fat and improves insulin sensitivity in older men with type 2 diabetes. Diabetes Care., 28: 662-667.

[26] Lim J. G., Kang H. J., Stewart K. J., 2004. Type 2 diabetes in Singapore: The role of exercise training for its prevention and management. Singapore Medical Journal., 45: 62-68.

[27] Eriksson M., Jhonson O., Boman K., Hallmans G., Hellsten G., Nilsson T. K. and Soderberg S., 2008. Improved fibrinolytic activity during exercise may be an effect of the adipocytederived hormones leptin and adiponectin. Thromb Res., 122 (5): 701-8.

[28] Saunders T. J., Palombella A., McGuire K. A., Janiszewski P. M, Despr'es J. P. and Ross R., 2012. Acute exercise increases adiponectin levels in abdominally obese men," Nutrition and Metabolism., 2012: 1-6.

[29] Oberbach A., Tönjes A., Klöting N., Fasshauer M., Kratzsch J., Busse M., Paschke R., Stumvoll M. and Blüher M., 2006. Effect of a 4 week physical training program on plasma concentration of inflammatory markers in patients with abnormal glucose tolerance. European Journal of Endocrinology., 154 (4): 577-585.

[30] Bluher M., Bullen J. W., Lee J. H., Kralisch S., Fasshauer M., Kloting M., Nie- bauer J., Schon M. R., Williams C., M. and Mantzoros C. S., 2006. Circulating adiponec- tin and expression of adiponectin receptors in human skeletal muscle: associations with metabolic parameters and insulin resistance and regulation by physical training. J Clin Endocrinol Metab; 91: $2310-2316$.

[31] Lehmann R., Kaplan V. and Bingisser R., 1997. Impact of physical activity on cardiovascular risk factors in IDDM. Diabetes Care., 20 (10): 1603-11.

[32] Baldi JC, Cassuto NA, Foxx-Lupo WT, et al 2011. Glycemic status affects cardiopulmonary exercise response in ath- letes with type I diabetes. Med Sci Sports Exerc 2011 Aug; 42 (8): 1454-9 84.

[33] Ismail I, Keating SE, Baker MK, et al 2012. A systematic review and meta-analysis of the effect of aerobic vs. resistance exercise training on visceral fat. Obes Rev 2012 Jan; 13 (1): 68-9186

[34] Bobbert T., Wegewitz U., Brechtel L. et al., 2007. Adiponectin oligomers in human serum during acute and chronic exercise: relation to lipid metabolism and insulin sensitivity," International Journal of Sports Medicine., 28 (1): $1-8$. 